\title{
Stimulation of indoleacetic acid production in a Rhizobium isolate of Vigna mungo by root nodule phenolic acids
}

Santi M. Mandal • Mahitosh Mandal · Amit K. Das •

Bikas R. Pati · Ananta K. Ghosh

Published online: 6 February 2009

(C) Springer-Verlag 2009

Erratum to: Arch Microbiol

DOI 10.1007/s00203-008-0455-6

Unfortunately, some of the authors' middle name was missed out in the author group of online published article. Instead of 'Santi Mandal, Mahitosh Mandal, Amit Das, Bikas Pati and Ananta Ghosh, it should read as 'Santi M. Mandal, Mahitosh Mandal, Amit K. Das, Bikas R. Pati and Ananta K. Ghosh'.

The online version of the original article can be found under doi:10.1007/s00203-008-0455-6.

S. M. Mandal · A. K. Das · A. K. Ghosh $(\bowtie)$

Department of Biotechnology,

Indian Institute of Technology Kharagpur, Kharagpur, India

e-mail: aghosh@hijli.iitkgp.ernet.in

S. M. Mandal · B. R. Pati

Department of Microbiology, Vidyasagar University,

Midnapur, India

M. Mandal

School of Medical Science and Technology,

Indian Institute of Technology Kharagpur, Kharagpur, India 